\title{
Intraoperative rupture of brainstem cavernous malformation
}

\author{
Case report
}

\author{
Gregory P. Lekovic, M.D., Ph.D., J.D., L. Fernando Gonzalez, M.D., \\ Vini G. Khurana, M.D., Ph.D., AND Robert F. SPetzler, M.D. \\ Division of Neurological Surgery, Barrow Neurological Institute, St. Joseph's Hospital \\ and Medical Center, Phoenix, Arizona
}

\begin{abstract}
$\checkmark$ Although cavernous malformations (CMs) are an important cause of intracranial hemorrhage, the natural history of these lesions is controversial. Both retrospective and prospective studies undertaken to define risk factors for hemorrhage from CMs have consistently identified the location of a lesion as a factor that has a significant impact on the rate of rupture, and brainstem CMs consistently have a higher rate of symptomatic hemorrhage than those at other locations. The mechanism underlying this disparity in rupture rates, however, remains obscure. Most authors attribute the difference, at least partially, to the sensitivity of the brainstem to hemorrhage. Regardless, the specific factors that cause a given CM to rupture are unknown.

The authors report their first encounter with an intraoperative rupture of a CM in the brainstem. This case underscores the risks encountered during the surgical approach to brainstem CMs and may provide insight into the pathophysiological mechanisms underlying the rupture of these lesions.
\end{abstract}

KEY WORDS • cavernous malformation • hemorrhage • venous malformation

A LTHOUGH CMs are an important cause of intracranial hemorrhage, their natural history is controversial. Estimates of the annual hemorrhage rate of these lesions vary from $0.25^{3}$ to $2.3 \%$ in retrospective analyses to more than $6.5 \%$ per patient per year in prospective studies. ${ }^{23}$ Both retrospective and prospective studies undertaken to define risk factors for hemorrhage from CMs have consistently identified the location of a lesion as a factor that has a significant impact on rate of rupture, and brainstem CMs consistently have a higher rate of symptomatic hemorrhage than those at other locations. Yet, the specific factors, if any, that cause a given CM to rupture are unknown. In addition, whether infratentorial CMs are more prone to hemorrhage than supratentorial ones or whether their ruptures are simply more evident because of the eloquence of the brainstem and posterior fossa is a matter of debate.

We report our first encounter with an intraoperative rupture of a CM in the brainstem. This case underscores the risks encountered during the surgical approach to brainstem CMs and may provide insight into the pathophysiological mechanisms underlying the rupture of these lesions.

\section{Case Report}

History and Examination. This 36-year-old man with a known CM of the midbrain tectum and superior medullary velum sought treatment for progressive headache, ataxia,

Abbreviations used in this paper: $\mathrm{CM}=$ cavernous malformation; $\mathrm{CT}=$ computed tomography. and dysarthria. On preoperative neuroimaging studies, the lesion measured $2.6 \times 1.8 \mathrm{~cm}$ (Fig. 1). The patient had previously undergone ventriculoperitoneal shunt placement at another hospital after his CM ruptured in 2002. He suffered two additional hemorrhages 1 and 4 months before presenting to our institution.

First Operation. The patient underwent an image-guided lateral supracerebellar infratentorial approach for resection of the brainstem CM. He was placed prone, with his head turned to the left and flexed. His topographic anatomy was registered to a StealthStation (Medtronic Sofamor Danek, Memphis, TN). A linear incision was made from the occipital protuberance to the level of the $\mathrm{C}$ 2 spinous process. A routine suboccipital craniotomy was then performed. The dura mater was opened using a semilunar incision with its base on the transverse sinus.

During the superficial lateral infratentorial dissection in the approach to the CM, a superficial cerebellar vein was inadvertently avulsed. Brisk bleeding was then encountered deep below the surgical site from a large venous lake along the tentorium in which the vein drained. The bleeding was controlled, but only after an estimated blood loss of approximately $1000 \mathrm{ml}$. Concomitantly, the contents of the posterior fossa began to swell and impede the surgical approach. The procedure was therefore aborted once control of the venous bleeding was obtained. The dura mater was tacked with interrupted suture and the bone flap was replaced.

First Postoperative Course. In the recovery room, the patient was obtunded, and a CT scan demonstrated hydrocephalus associated with a fourth ventricular hemorrhage 

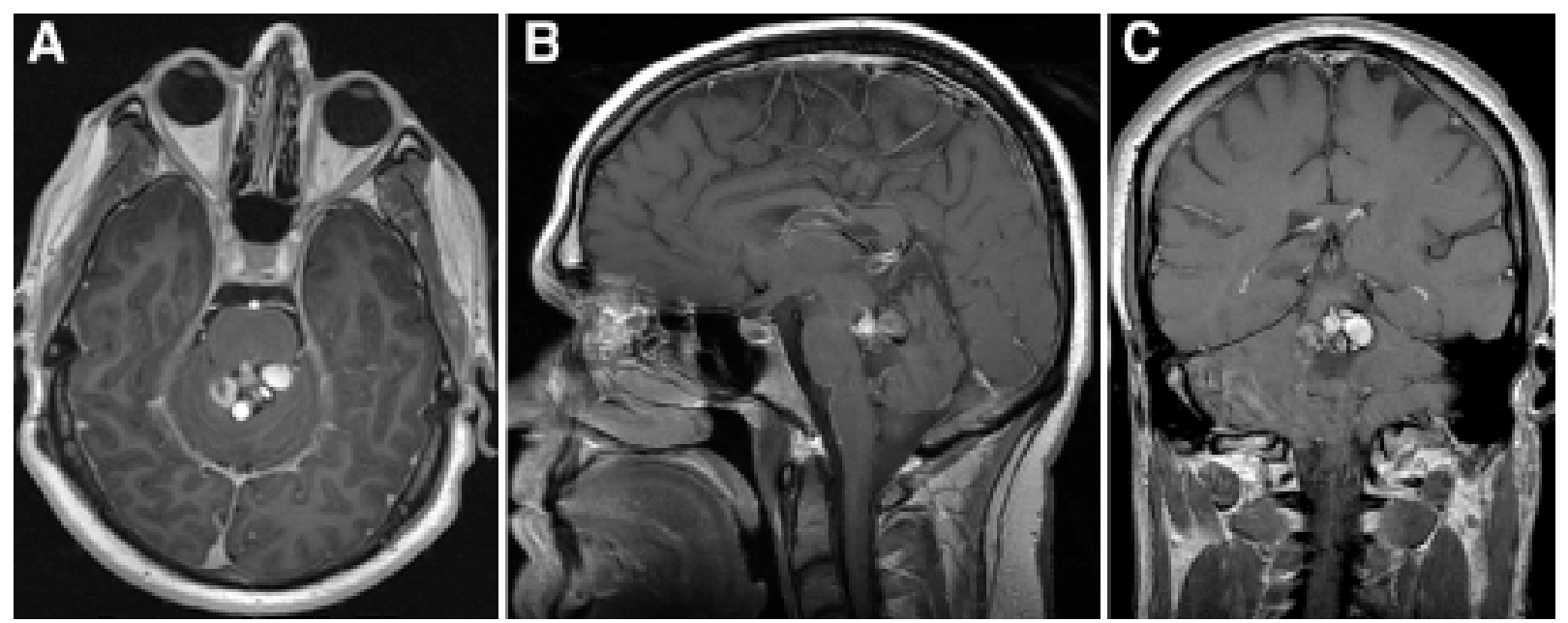

FIG. 1. Preoperative axial (A), sagittal (B), and coronal (C) gadolinium-enhanced magnetic resonance (MR) images revealing a lesion in the superior medullary velum. The heterogeneous signal intensity is consistent with blood products of mixed ages. There is no contrast enhancement.

(Fig. 2). An external ventricular drain was placed, and the patient was admitted to the intensive care unit, where his neurological status improved. He followed commands bilaterally, his pupils were equal, and his motor examination revealed equal strength bilaterally without drift. On postoperative Day 2, he was extubated. After extubation, the patient suffered a seizure and his neurological status declined precipitously. He was reintubated and a loading dose of fosphenytoin was administered, which controlled his seizure. Neurology and pulmonary medicine consultations were obtained. The patient's condition stabilized.

Second Operation. Four days after the initial attempt at resection (Fig. 3), the patient was returned to the operating room to reopen the suboccipital craniotomy and resect the CM. He was positioned as for the first procedure, and the incision was reopened. When the dura mater was opened, infarcted cerebellar hemisphere immediately herniated through the bone defect. Because of the tightness of the posterior fossa contents, the superior cerebellar hemisphere had to be resected to gain access to the CM. The lesion was resected in its entirety without incident (Fig. 4), as was a large intralesional blood clot. There was no evidence of extralesional or fourth ventricular hemorrhage. Although no venous angioma was evident on preoperative studies, abnormal veins consistent with a venous malformation were identified at surgery. Blood loss was estimated at $500 \mathrm{ml}$. Due to swelling of the posterior fossa, the bone flap was not replaced and the cerebellar hemisphere was decompressed.

Second Postoperative Course. Postoperatively, the patient was readmitted to the intensive care unit. He had mild hemiparesis (4/5) on the left and no gag reflex. Otherwise, he was neurologically intact. The patient had another seizure, which required multiagent therapy to control. The remainder of his hospital course was marked by respiratory failure related to streptococcal pneumonia. Seven days after the second operation, he was extubated and successfully weaned from his ventriculostomy. A modified barium swallow test failed and the patient required percutaneous gastrostomy for feeding. On postop- erative Day 9, a follow-up CT scan (Fig. 5) demonstrated improvement in the posterior fossa swelling with expected postoperative findings, and the patient was transferred to acute inpatient rehabilitation. At discharge, his seizures were controlled with dual-agent antiepileptic therapy.

On his final follow-up evaluation performed at 3 months, the patient reported persistent but improving diplopia and dysphagia. On examination, a left internuclear ophthalmo-

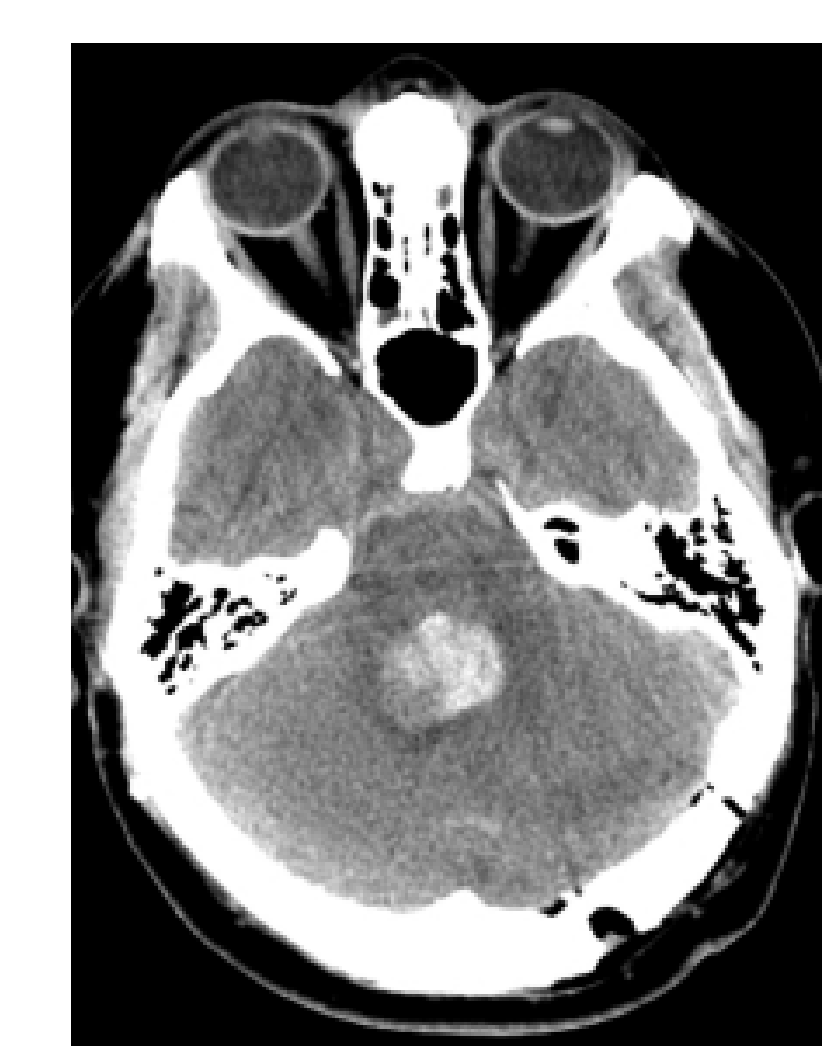

FIG. 2. A CT scan of the head obtained immediately postoperatively without contrast material, demonstrating acute blood in the fourth ventricle. 


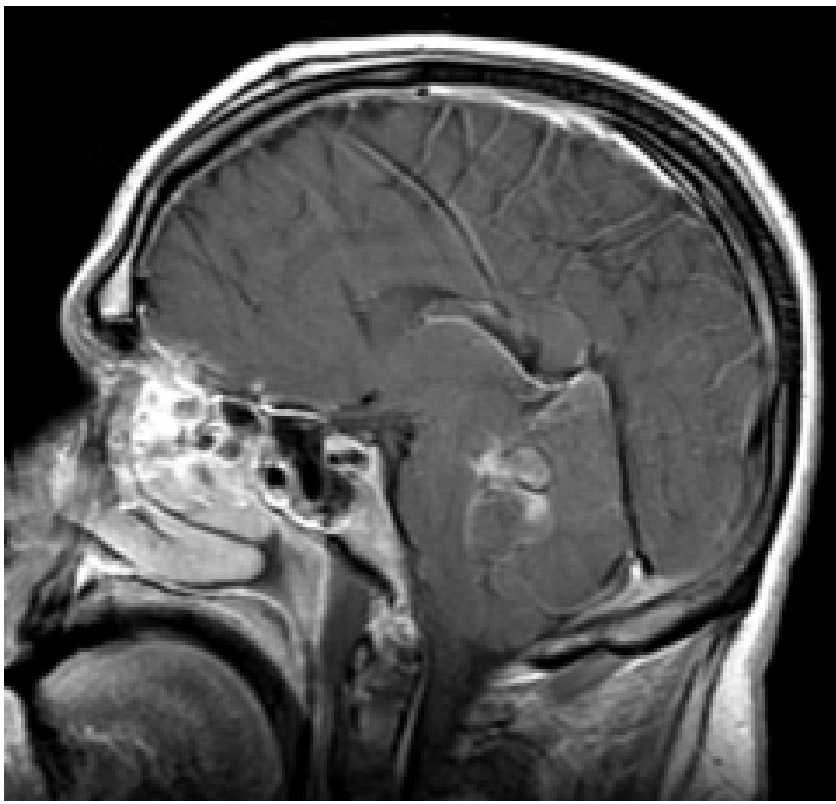

FIG. 3. Sagittal MR image obtained before the second surgery, demonstrating continuous extension of hemorrhage from the lesion into the fourth ventricle.

plegia, left facial weakness, and left dysmetria were found. Otherwise, he was neurologically intact.

\section{Discussion}

The natural history of CMs remains controversial. Whether rupture rates are assessed retrospectively or prospectively, and whether familial and brainstem CMs are subsets of these lesions that should be considered separately are issues that have an impact on the natural history of these malformations.

Brainstem lesions account for approximately $20 \%$ of all intracranial CMs; the most common site within the brainstem is the pons. ${ }^{15,16,18}$ Unlike supratentorial lesions, brain- stem CMs are less likely to manifest with seizure and more likely to do so with symptoms related to mass effect such as cranial neuropathy, hemiparesis, or hydrocephalus. A history of multiple hemorrhages on presentation is not uncommon. ${ }^{16,18}$ Because of these lesions' different presentations and their higher apparent rupture rate compared with supratentorial CMs, some authors have argued that brainstem lesions should be considered distinct entities from supratentorial ones. ${ }^{15}$

In retrospective studies of all intracranial CMs, based on the assumption that lesions are present at birth, the most commonly reported rates of hemorrhage vary from 0.25 to $2.3 \%, 3,9$ although rates as high as $60 \%$ have been reported for brainstem CMs. ${ }^{14}$

Several factors have been proposed to predispose a CM to rupture, including lesion location, ${ }^{3,9,10,15}$ a history of rupture, size, ${ }^{8,12}$ and the presence of an associated developmental venous anomaly. ${ }^{1}$ The factor most consistently associated with increased risk of rupture across series is location. The mechanism for such a disparity in rupture rates, however, remains obscure, and most authors attribute this difference at least in part to the sensitivity of the brainstem to hemorrhage. In addition, a previous rupture is associated strongly in the literature with as much as a sevenfold increase in the risk of prospective rupture..$^{10,13}$

This case raises an interesting question about the relationship among CMs, venous anomalies, and normal venous drainage. Some authors have attempted to link the presence or absence of an associated venous malformation with a higher rate of rupture. ${ }^{1}$ Others have proposed that venous malformations, when seen in association with CMs, are the "actual pathological lesion" that precipitates formation of CMs and hemorrhage. ${ }^{22}$ The latter authors further recommend excision of associated venous malformations to prevent recurrence of the CM. In our experience, ${ }^{4,17}$ however, CMs have universally been associated with venous anomalies. Moreover, we have found that the venous component of the malformation must be preserved to maintain normal venous drainage and to avoid venous infarction. ${ }^{4}$

In the present case, the vein we coagulated was located
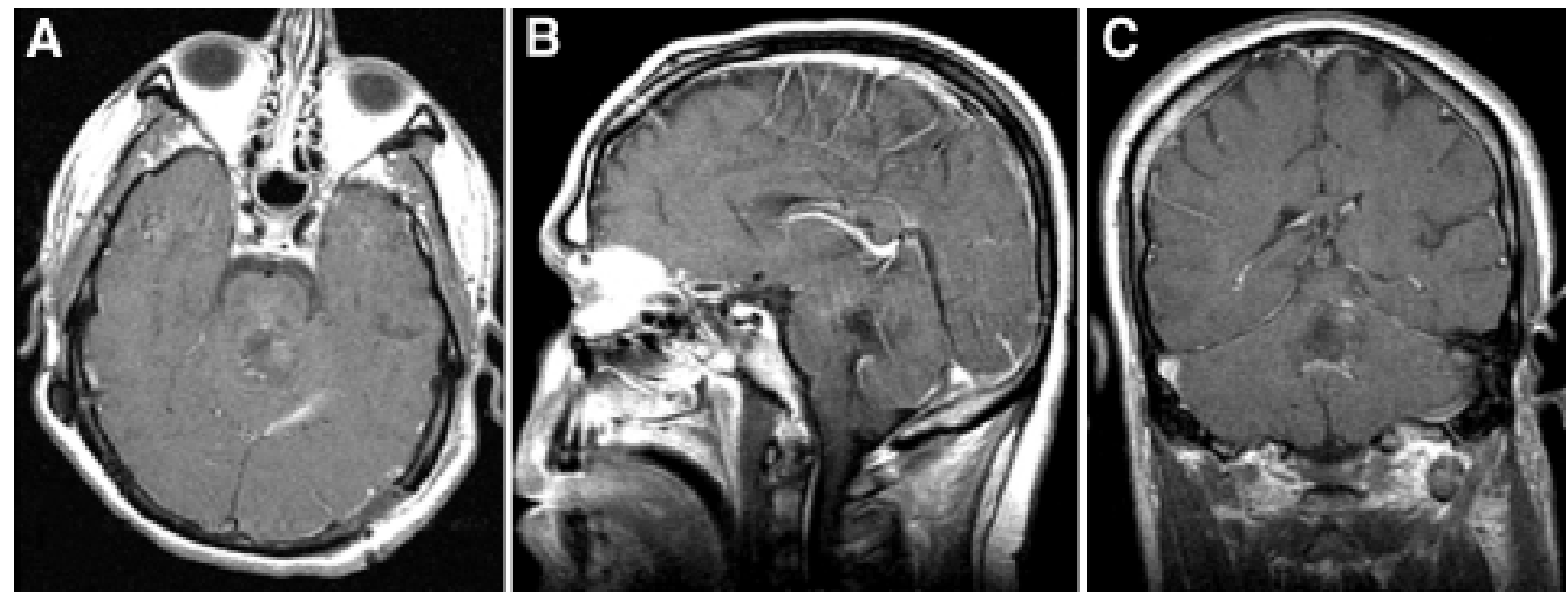

FIG. 4. Axial (A), sagittal (B), and coronal (C) MR images obtained after the second surgery, demonstrating complete extirpation of the lesion and associated acute blood components. 


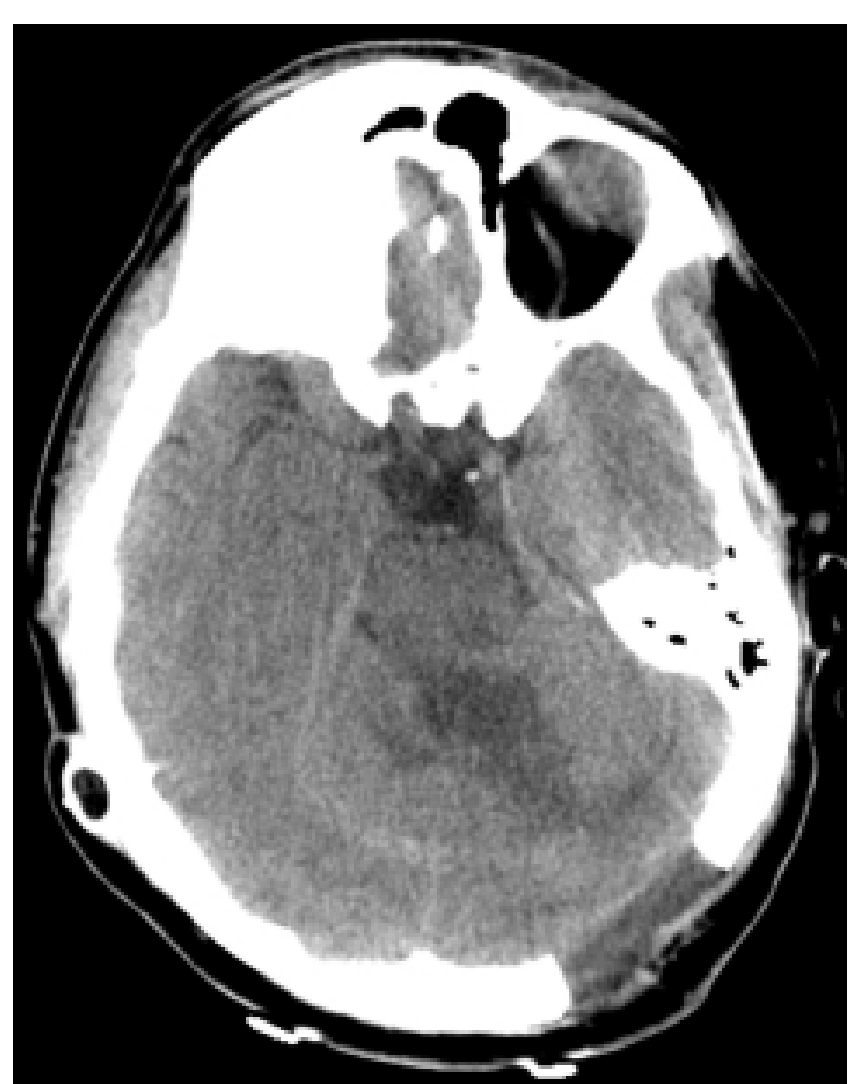

FIG. 5. A CT scan of the head obtained without contrast material before the patient was discharged, demonstrating improvement of posterior fossa swelling with effaced but patent basal cisterns.

over the superior aspect of the lateral cerebellar hemisphere, and was therefore remote from the CM and associated venous malformation. Nevertheless, the possibility remains that this vein contributed to the drainage of the malformation. If so, the change in venous hemodynamics caused by its obliteration may explain the occurrence of intraoperative rupture. We have previously shown that the intraluminal pressure of venous angiomas associated with CMs is identical to dural sinus pressure, even when venous angiomas appear to drain sluggishly on angiographic studies. ${ }^{17}$ Thus, it is plausible that general changes in cerebral venous hemodynamics may be transmitted to a venous malformation associated with a CM. However, the way in which changes in venous malformation hemodynamics might increase the chance of hemorrhage from a $\mathrm{CM}$ is unclear.

To date, there is no convincing histological or ultrastructural evidence of direct communication between a $\mathrm{CM}$ and an associated venous anomaly. An anecdotal report $^{19}$ of gross connections between a CM and its associated venous anomaly is at odds with our observations during routine excision of $\mathrm{CMs}$, in which we leave their associated venous malformation intact. Moreover, in a study in which investigators compared the histological and ultrastructural anatomy of unruptured and hemorrhagic CMs, they failed to document direct evidence of a communication or continuity between normal-appearing veins and a CM. ${ }^{21}$ These authors found that all CMs demon- strated incompetent basement membranes. The endothelial cells of hemorrhagic CMs were more likely to have large intraluminal vesicles and absent tight junctions. They proposed that the mechanism of rupture of CMs is related to extravasation of blood across the endothelium of the sinusoidal channels composing the lesion. Although this concept is consistent with an increased likelihood of hemorrhage from a CM with changes in venous pressure or filling dynamics, the structural basis by which changes in venous pressure would be transmitted to the lesion to precipitate the hemorrhage remains unknown.

Finally, although multiple studies have unequivocally demonstrated the benefit of surgery for these lesions, $2,5,7,11$, ${ }^{16,20}$ our experience in this case emphasizes the importance of thoughtfully aborting a procedure when this action is dictated by intraoperative events. Once a patient stabilizes, complete resection of the malformation can be pursued. In the absence of fourth ventricular hemorrhage, we would have preferred to wait longer before reoperation. Some authors have argued that early surgery for brainstem CMs after rupture is important to minimize the development of perilesional gliosis. ${ }^{6}$ Nevertheless, Samii, et al., ${ }^{18}$ failed to find a statistical significance in outcome in lesions that were surgically treated either within or after 3 months of rupture in a series of 36 brainstem CMs. Our preference has been to allow, when possible, sufficient time for the clot to liquefy and thereby assist in the dissection of the malformation from the surrounding brainstem. However, this patient's brainstem CM had ruptured three times (including the intraoperative rupture) within 3 months, indicating that it was extremely fragile, and we feared repeated rupture. Most importantly, the patient was symptomatic from his fourth ventricular hemorrhage and required decompression of the fourth ventricle.

\section{Conclusions}

This is the first instance of intraoperative rupture of a brainstem CM in the experience of the senior author (R.F.S.) in resecting more than 200 of these lesions. Moreover, we are unaware of any other reports of this phenomenon. Although it is impossible to ascertain the specific cause of the intraoperative rupture, the temporal association among the venous hemorrhage encountered during surgery, its obliteration, and the rupture support the suggestion that alterations in venous hemodynamics caused by coagulation of superficial cerebellar venous drainage precipitated rupture of this fragile CM.

\section{References}

1. Abdulrauf SI, Kaynar MY, Awad IA: A comparison of the clinical profile of cavernous malformations with and without associated venous malformations. Neurosurgery 44:41-47, 1999

2. Bertalanffy H, Gilsbach JM, Eggert HR, Seeger W: Microsurgery of deep-seated cavernous angiomas: report of 26 cases. Acta Neurochir (Wien) 108:91-99, 1991

3. Del Curling O Jr, Kelly DL Jr, Elster AD, Craven TE: An analysis of the natural history of cavernous angiomas. J Neurosurg 75:702-708, 1991

4. Detwiler PW, Porter R, Lawton MT, Spetzler RF: Detection of delayed cerebral vasospasm, after rupture of intracranial aneurysms, by magnetic resonance angiography. Neurosurgery 41:997-998, 1997 


\section{Intraoperative rupture of a brainstem cavernous malformation}

5. Fahlbusch R, Strauss C, Huk W: Pontine-mesencephalic cavernomas: indications for surgery and operative results. Acta Neurochir Suppl 53:37-41, 1991

6. Fahlbusch R, Strauss C, Huk W, Rockelein G, Kompf D, Ruprecht KW: Surgical removal of pontomesencephalic cavernous hemangiomas. Neurosurgery 26:449-457, 1990

7. Ferroli P, Sinisi M, Franzini A, Giombini S, Solero CL, Broggi G: Brainstem cavernomas: long-term results of microsurgical resection in 52 patients. Neurosurgery 56:1203-1214, 2005

8. Hasegawa T, McInerney J, Kondziolka D, Lee JYK, Flickinger JC, Lunsford LD: Long-term results after stereotactic radiosurgery for patients with cavernous malformations. Neurosurgery 50:1190-1198, 2002

9. Kim DS, Park YG, Choi JU, Chung SS, Lee KC: An analysis of the natural history of cavernous malformations. Surg Neurol 48:9-17, 1997

10. Kondziolka D, Lunsford LD, Kestle JRW: The natural history of cerebral cavernous malformations. J Neurosurg 83: 820-824, 1995

11. Konovalov A, Samii M, Porter RW, Spetzler RF, Houteville JP, Yoshimoto T, et al: Brainstem cavernoma. Surg Neurol 54: 418-421, 2000

12. Kupersmith MJ, Kalish H, Epstein F, Yu G, Berenstein A, Woo $\mathrm{H}$, et al: Natural history of brainstem cavernous malformations. Neurosurgery 48:47-54, 2001

13. Mathiesen T, Edner G, Kihlström L: Deep and brainstem cavernomas: a consecutive 8-year series. J Neurosurg 99:31-37, 2003

14. Mizoi K, Yoshimoto T, Suzuki J: Clinical analysis of ten cases with surgically treated brain stem cavernous angiomas. Tohoku J Exp Med 166:259-267, 1992

15. Moriarity JL, Clatterbuck RE, Rigamonti D: The natural history of cavernous malformations. Neurosurg Clin N Am 10: 411-417, 1999
16. Porter RW, Detwiler PW, Spetzler RF, Lawton MT, Baskin JJ, Derksen PT, et al: Cavernous malformations of the brainstem: experience with 100 patients. J Neurosurg 90:50-58, 1999

17. Rigamonti D, Spetzler RF: The association of venous and cavernous malformations. Report of four cases and discussion of the pathophysiological, diagnostic, and therapeutic implications. Acta Neurochir (Wien) 92:100-105, 1988

18. Samii M, Eghbal R, Carvalho GA, Matthies C: Surgical management of brainstem cavernomas. J Neurosurg 95:825-832, 2001

19. Sasaki O, Tanaka R, Koike T, Koide A, Koizumi T, Ogawa H: Excision of cavernous angioma with preservation of coexisting venous angioma. Case report. J Neurosurg 75:461-464, 1991

20. Steinberg GK, Chang SD, Gewirtz RJ, Lopez JR: Microsurgical resection of brainstem, thalamic, and basal ganglia angiographically occult vascular malformation. Neurosurgery 46: 260-271, 2000

21. Tu J, Stoodley MA, Morgan MK, Storer KP: Ultrastructural characteristics of hemorrhagic, nonhemorrhagic, and recurrent cavernous malformations. J Neurosurg 103:903-909, 2005

22. Wurm G, Schnizer M, Fellner FA: Cerebral cavernous malformations associated with venous anomalies: surgical considerations. Neurosurgery 57 (1 Suppl):42-58, 2005

23. Zabramski JM, Wascher TM, Spetzler RF, Johnson B, Golfinos J, Drayer BP, et al: The natural history of familial cavernous malformations: results of an ongoing study. J Neurosurg 80: 422-432, 1994

Manuscript received April 14, 2006.

Accepted in final form May 30, 2006.

Address reprint requests to: Robert F. Spetzler, M.D., c/o Neuroscience Publications, Barrow Neurological Institute, 350 West Thomas Road, Phoenix, Arizona 85013. email: neuropub@chw.edu. 